\title{
A GESTÃO DO CONHECIMENTO NA TRANSFORMAÇÃO DIGITAL PARA A INDÚSTRIA 4.0: TECNOLOGIAS DIGITAIS E SUAS APLICAÇÕES EM SETORES ECONÔMICOS
}

\author{
KNOWLEDGE MANAGEMENT IN DIGITAL TRANSFORMATION FOR INDUSTRY 4.0: \\ DIGITAL TECHNOLOGIES AND THEIR APPLICATIONS IN ECONOMIC SECTORS
}

Recebido em: 12 dez. 2020

Aprovado em: 20 mar. 2021

Versão do autor aceita publicada online: 20 mar. 2021

Publicado online: 30 jun. 2021

Como citar esse artigo - American Psychological Association (APA):

Senna, D. A., \& Ribeiro, J. S. de A. N. (2023, jan./mar). A gestão do conhecimento na transformação digital para a Indústria 4.0: tecnologias digitais e suas aplicações em setores econômicos. Exacta, 21(1), 224-248. https://doi.org/10.5585/exactaep.2021.18917

Submeta seu artigo para este periódico $\beta$

Processo de Avaliação: Double Blind Review

Editor: DDr. Luiz Fernando Rodrigues Pinto

Dados Crossmark 
A GESTÃO DO CONHECIMENTO NA TRANSFORMAÇÃO DIGITAL PARA A INDÚSTRIA 4.0: TECNOLOGIAS DIGITAIS E SUAS APLICAÇÕES EM SETORES ECONÔMICOS

\author{
KNOWLEDGE MANAGEMENT IN DIGITAL TRANSFORMATION FOR INDUSTRY 4.0: DIGITAL \\ TECHNOLOGIES AND THEIR APPLICATIONS IN ECONOMIC SECTORS
}

\author{
(D) Diego Augustus Senna ${ }^{1}$ \\ (iD) Jurema Suely de Araújo Nery Ribeiro²
}

Resumo: A transformação digital, impulsionada pela Indústria 4.0, promove ambiente volátil e competitivo e acelera o desenvolvimento de inovadoras tecnologias, por vezes incompatíveis com práticas tradicionais. Organizações precisam, então, reformular seu capital intelectual, o que envolve questões de gestão e compartilhamento do conhecimento. Este trabalho busca analisar, por meio de revisão sistemática e bibliometria, quais tecnologias e setores econômicos tendem a estar associados a essas questões, utilizando-se categorização de artigos, análises estatísticas e uma nuvem de palavras. Identificou-se que há crescimento exponencial do número de pesquisas - decorrente do aumento de competitividade - e que o setor acadêmico é predominante, seguido pelo setor de manufatura. Os contextos tecnológicos são muito variáveis, mas predominantemente baseados em tecnologias de informação e comunicação, pilares da Indústria 4.0. Compreender onde as tecnologias digitais - cada vez mais relevantes - estão sendo aplicadas é fundamental para os setores público e privado. Esta pesquisa pode futuramente ser expandida, contemplando novos trabalhos.

Palavras-chave: transformação digital. indústria 4.0. gestão e compartilhamento do conhecimento. tecnologias digitais. bibliometria.

Abstract: The digital transformation, driven by Industry 4.0, promotes a volatile and competitive environment and accelerates the development of innovative technologies, sometimes incompatible with traditional practices. It is necessary for organizations to reshape their intellectual capital, considering issues of knowledge management and sharing. This article seeks to analyze, through systematic review and bibliometrics, which technologies and economic sectors tend to be associated with these issues, using categorization of papers, statistical analyses and a word cloud. The results show that there is an exponential growth in number of researches - due to increased competitiveness - and that the academic sector is predominant, followed by the manufacturing sector. There is a lot of variation considering technological contexts, but information and communication technologies, pillars of Industry 4.0, are predominant. Understanding where digital technologies - increasingly relevant - are being applied is fundamental for public and private backgrounds. This research may be expanded in the future, including new papers.

Keywords: digital transformation. industry 4.0. knowledge management and sharing. digital technologies. bibliometrics.

\footnotetext{
${ }^{1}$ Mestre em Saneamento, Meio Ambiente e Recursos Hídricos / Universidade Federal de Minas Gerais - UFMG - Belo Horizonte, MG - Brasil augustus.senna@yahoo.com.br

2 Doutora em Sistemas de Informação e Gestão do Conhecimento / Universidade FUMEC - Belo Horizonte, MG - Brasil jurema.nery@gmail.com
} 


\section{Introdução}

No mundo globalizado, o mercado é caracterizado por níveis crescentes de volatilidade e competitividade. Práticas antes consideradas eficientes e vencedoras devem ser constantemente suprimidas e substituídas por novos padrões, balizados por tecnologias inovadoras que surgem a todo momento. Esse contexto de transformação, muitas vezes associado diretamente às indústrias manufatureiras, atinge os mais diversos setores da economia, de forma direta ou indireta, e afeta a cultura das organizações.

Muitas das tecnologias responsáveis por essa "transformação digital", como é definida, já existem no mercado há algum tempo. Não havia, porém, contexto de tamanha pressão que promovesse sua aplicação conjunta visando o ganho de competitividade. O interesse em criar ambiente propício e conectado para explorar o potencial de tantas inovações consolidou-se no ato do lançamento, em 2013, da Plataforma "Industrie 4.0" pelo governo alemão. A Quarta Revolução Industrial representa, conforme apontado por Kagermann, Anderl, Gausemeier, Schuh and Wahlster (2016), profunda ruptura - ou paradigma de mudança - com as relações econômicas tradicionais. A digitalização de todos os estágios da atividade produtiva é necessária e, portanto, as tecnologias essenciais para cada organização são variáveis. Contudo, tecnologias de informação e comunicação (TICs) são sempre ponto de central interesse (Poloskov, Zheltenkov, Braga, \& Kuznetsova, 2020).

Nesse cenário, a gestão e o compartilhamento do conhecimento são profundamente afetados: intensas mudanças organizacionais se estendem ao longo das cadeias de suprimentos. As empresas devem se reinventar, e a base fundamental para qualquer transformação cultural bem sucedida é o conhecimento. Existe relação de mão dupla: por um lado, a implementação de tecnologias e processos é dependente da capacidade de absorver e aplicar novos conhecimentos. Por outro, a gestão desses mesmos conhecimentos pode precisar de completa alteração para se adaptar aos novos contextos tecnológicos. Em ambos os cenários, dificuldades surgem, e justifica-se, portanto, a necessidade de identificar os contextos tecnológicos - conjuntos de tecnologias direcionadas a objetivos comuns bem definidos, como o Digital Learning - e setores econômicos mais impactados para desenvolver ações que incentivem a inovação dentro e fora das organizações (Maglio, 2021).

Este artigo busca explorar, em pesquisas, quais tecnologias e contextos tecnológicos, principalmente relacionados à Indústria 4.0, estão sendo associados a questões de gestão e compartilhamento do conhecimento, em ambiente cercado por desafios, e avaliar quais setores econômicos tendem a estar envolvidos. Cinco hipóteses norteadoras, objetivando-se direcionar o estudo dos dados, foram formuladas com base na literatura apresentada: i) o aumento de competitividade do mercado eleva, cada vez mais, o interesse por pesquisas na área; ii) diante da necessidade de constante aperfeiçoamento profissional, relações com o setor acadêmico são 
predominantes; iii) apesar dos impactos serem influentes em todas as esferas da economia, o setor de manufatura assume papel central; iv) considerando o forte embasamento da Quarta Revolução Industrial em TICS, estas são abordadas mais frequentemente; v) as tecnologias consolidadas em cada setor econômico são muito variáveis.

Este artigo encontra-se organizado em cinco seções. O item 2 corresponde à exposição do referencial teórico de embasamento da pesquisa. A metodologia, de caráter exploratório e bibliométrico, está apresentada na seção 3. Os resultados, quantificados em gráficos e tabelas e apoiados por inferências qualitativas, são demonstrados e discutidos na seção 4. Por fim, as considerações finais estão dispostas no item 5 .

\section{Referencial Teórico}

Esta seção encontra-se dividida em duas subseções: Transformação Digital (2.1) e Gestão e Compartilhamento do Conhecimento (2.2).

\subsection{Transformação Digital}

A transformação digital, impulsionada pela Indústria 4.0, é marcada por forte presença de TICs, existindo grande circulação de dados. Há necessidade de constante conexão entre as empresas e atores externos das cadeias de valor, bem como entre os próprios processos internos. Ou seja, realizar a transformação digital significa digitalizar todos os nós finais presentes no setor de atuação de uma organização (Kagermann et al., 2016). Embora a Indústria 4.0 tenha sido iniciada na Alemanha, diversos países rapidamente lançaram seus próprios programas, buscando aumentar a competitividade de suas organizações. Ao analisar a iniciativa americana, Smart Manufacturing, sul-coreana, Smart Factory Program, ou a de qualquer outro país, a centralidade do uso de dados digitais é sempre o ponto central de convergência (Lenz, Wuest, \& Westkämper, 2018).

Com o aumento da personalização e da complexidade dos produtos e serviços, visando diferenciação no mercado, os tradicionais sistemas de automação tornaram-se rapidamente obsoletos (Gorecky, Khamis, \& Mura, 2017). A transformação digital é fundamental para garantir flexibilidade de processos e produção, enquanto simultaneamente aprimora a eficiência e a produtividade. Contudo, depende fortemente da união de informações, recursos e pessoas em um ambiente comum (Klitou, Conrads, \& Rasmussen, 2017). Nesse contexto, as soluções digitais podem ser aplicadas para uma infinidade de objetivos e, consequentemente, sua modelagem e implementação são muito variáveis. Podem ser apontadas como soluções de grande importância aquelas diretamente relacionadas às TICs, como, por exemplo, os Sistemas Cyber-Físicos (Cyber Phisical Systems - CPS), a Internet das Coisas 
(Internet of Things - IoT), a Computação em Nuvem (Cloud Computing) e o Big Data (Gölzer \& Fritzche, 2017).

O programa alemão da Industrie 4.0 objetiva fornecer know-how a diversos grupos de características muito distintas, abrangendo pequenas, médias e grandes empresas, assim como microempresários particulares. Atinge, também, outros domínios, atraindo, por exemplo, representantes do setor acadêmico, cientistas sociais, políticos e administradores. A propagação de conhecimentos e a adoção de tecnologias, contudo, não ocorre com a mesma velocidade em todos os setores, embora a universalização seja desejada (Klitou et al., 2017).

\subsection{Gestão e Compartilhamento do Conhecimento}

O conhecimento é um recurso de fundamental importância para a formação das relações de poder e competitividade entre organizações. Diversos autores já alertavam para essa contextualização desde os anos 1990. Toffler (1990) observou que o conhecimento deixou de ser mero auxiliar do poder monetário e da força física para se tornar sua própria essência. Drucker (1994) definiu que o conhecimento seria a matéria-prima do futuro, considerando a sociedade moderna e suas incessantes transformações, e anunciou a formação de uma "sociedade do conhecimento". Essa visão foi posteriormente reforçada por outros autores (Choo, 2003; Davenport \& Prusak, 2003; Nonaka \& Takeuchi, 1997). Na era da globalização, o conhecimento embutido nos produtos e serviços pode ser definido como o único caminho para vencer empresas concorrentes e sobreviver (Belluzzo \& Feres, 2009).

A Gestão do Conhecimento (GC) utiliza o conhecimento organizacional - em termos de habilidades individuais, competências, pensamentos, inovações e ideias - para melhorar a eficiência e a efetividade de uma organização (Centenaro, Bonemberger, \& Laimer, 2016). As organizações que têm as melhores informações ou que as controlam de forma mais eficaz são dominantes. E, com base no uso do conhecimento de forma integrada e colaborativa, é preciso focar em criação de valor não apenas internamente, mas considerando toda a cadeia de fornecimento (Revilla \& Knoppen, 2015). Nesse contexto, as TICs são fundamentais.

As tecnologias sempre atuaram como pilar de sustentação da GC, uma vez que são representadas como estruturas teóricas de sistematização (Batista, 2012; Davenport \& Prusak, 2003). Contudo, os recentes avanços tecnológicos acentuaram essa relação, tendo em vista que implicam em novas interações, promovendo apoio aos processos de aplicação e compartilhamento do conhecimento e surgindo como fontes de informação (Ribeiro, França, Corrêa, \& Ziviani, 2019). Para alcançar o sucesso, não basta que a organização tenha o simples acesso às tecnologias, uma vez que, cedo ou tarde, os concorrentes também o terão. O diferencial está em saber usá-las, aplicando-as de maneira a 
realmente adquirir diferenciais competitivos. Justifica-se, portanto, a importância de compreender a ligação entre as tecnologias e a GC.

\section{Metodologia}

Este trabalho é parte de uma pesquisa desenvolvida entre os meses de abril e maio de 2020. Foram buscados, em quatro bases de dados de artigos científicos, estudos sobre a temática proposta, conforme a descrição apresentada no Quadro 1.

A metodologia, baseada em revisão sistemática de literatura (RSL), apresenta caráter bibliométrico, uma vez que objetiva, a partir de exploração preliminar, definir indicadores para identificar e quantificar temas e tendências no material estudado (Rodrigues, Tavar, Nogueira, \& Librelotto, 2016). O modelo de quatro etapas utilizado por Araújo, Carneiro and Palha (2020) forneceu embasamento para a construção da amostra e o delineamento das análises:

1. Identificação: por meio de palavras-chave, trabalhos são buscados em bases de dados científicas;

2. Filtragem: critérios de exclusão, como repetição, tipo de documento e idioma, são aplicados para definir a amostra inicial;

3. Elegibilidade: através da leitura dos títulos e resumos, estudos que não apresentam conexão com a temática são localizados e excluídos da amostra;

4. Inclusão: uma matriz de análise é criada para avaliar o material selecionado. Mediante leitura dos artigos, temas e pontos relevantes são identificados, registrados, quantificados e investigados. 


\section{Quadro 1}

\section{Descrição metodológica}

\begin{tabular}{|c|c|}
\hline Critério & Descrição \\
\hline $\begin{array}{l}\text { Descritores } \\
\text { pesquisados }\end{array}$ & $\begin{array}{l}\text { A expressão utilizada foi composta por três termos, unidos pelo operador "AND": } \\
\text { 1. Título, resumo ou palavras-chave: } \\
\text { a) "digital transformation" OR "digital transformations" OR "transformação digital" } \\
\text { OR "transformações digitais" OR "digital technology" OR "digital technologies" } \\
\text { OR "tecnologia digital" OR "tecnologias digitais"; } \\
\text { b) "knowledge management" OR "gestão do conhecimento" OR "shared } \\
\text { knowledge" OR "conhecimento compartilhado" OR "knowledge sharing" OR } \\
\text { "sharing knowledge" OR "compartilhamento do conhecimento" OR "knowledge } \\
\text { share" OR "share knowledge" OR "compartilhar conhecimento"; } \\
\text { 2. Todo o documento: "challenge" OR "challenges" OR "desafio" OR "desafios". }\end{array}$ \\
\hline Categoria & Artigos científicos publicados em periódicos. \\
\hline Idioma & Qualquer (sem restrição). \\
\hline Ano & Qualquer (sem definição de intervalo temporal, uma vez que o assunto é recente). \\
\hline Bases de dados & Scielo, Science Direct, Scopus, Spell. \\
\hline Exclusão & Por repetição ou falta de aderência à temática. \\
\hline Contexto & $\begin{array}{l}\text { Setores econômicos que enfrentam desafios e detectam oportunidades para a gestão e o } \\
\text { compartilhamento do conhecimento, considerando as tecnologias impulsionadoras da } \\
\text { transformação digital no contexto da Indústria 4.0. }\end{array}$ \\
\hline Justificativa & $\begin{array}{l}\text { A Indústria } 4.0 \text { corresponde à implementação de diversas tecnologias digitais interligadas em } \\
\text { uma mesma conjuntura. Os impactos são promovidos e sentidos em diversos setores. } \\
\text { Contudo, nem todas as áreas da economia são impactadas da mesma forma e ao mesmo } \\
\text { tempo, e a importância dada a cada tecnologia pode variar. }\end{array}$ \\
\hline
\end{tabular}

Fonte: Autores (2021).

A transformação digital e a gestão e o compartilhamento do conhecimento são os elementos estruturais deste trabalho e por isso foram buscados no título, no resumo ou nas palavras-chave, restringindo-se os resultados, conforme descrito no Quadro 1. Os desafios foram buscados ao longo do texto, uma vez que objetivou-se explorar todo o conteúdo dos documentos em busca de dificuldades relatadas, bem como os setores econômicos e contextos tecnológicos envolvidos.

A base de dados Scopus apresentou-se como a mais importante fonte de referências para este trabalho, uma vez que indicou 45 resultados, sendo um descartado por não se enquadrar na categoria de artigo científico. A amostra foi complementada pela busca executada na plataforma Science Direct, que retornou 9 resultados, dos quais 4 foram adicionados à análise e 5 foram descartados por repetição, uma vez que já haviam sido encontrados na busca da Scopus. Scielo e Spell não indicaram resultados.

O software LibreOffice Calc foi utilizado para a categorização dos 48 trabalhos da amostra resultante. Cada artigo foi identificado por um número, sendo explorados e registrados os seguintes metadados: autores, título, ano de publicação, periódico, volume, número, intervalo de páginas, idioma, palavras-chave e área temática definida pelo sistema SciVal (apenas para estudos provenientes da plataforma Scopus).

Neste trabalho, foram identificados e quantificados, para análise, o setor econômico a que cada artigo se refere, assim como a principal tecnologia ou contexto tecnológico da transformação digital, 
considerando os pilares da Indústria 4.0. A classificação, apresentada no Quadro 2 - referente aos setores econômicos - e no Quadro 3 - referente às tecnologias - foi elaborada após análise preliminar, tomando como embasamento o próprio conteúdo dos artigos e sua relação com aplicações típicas da Quarta Revolução Industrial. Embora alguns estudos apresentem discussões referentes a mais de uma tecnologia ou setor econômico, foram considerados apenas os elementos centrais, associados diretamente à estrutura e aos objetivos.

Existe, na literatura, grande variação na identificação de tecnologias componentes da Indústria 4.0. Há, contudo, frequente aderência - direta ou indireta - àquelas listadas no Quadro 3. TICs e loT são pontos em comum entre as diversas análises. Polat and Erkollar (2021), por exemplo, mencionam também Big Data, Inteligência Artificial e Computação em Nuvem, além de ressaltarem incisivamente a importância do fator humano e das diversas formas de comunicação. Maglio (2021) adiciona Robótica, Veículos Autônomos e Drones, Realidade Virtual, Blockchain, Rastreabilidade Digital e Manufatura Aditiva (Impressão 3D). Poloskov et al. (2020), por sua vez, acrescentam Sistemas Cyber-Físicos (CPS), Realidade Aumentada, Modelagem 3D e Integração Horizontal e Vertical de Sistemas.

Análises foram desenvolvidas para identificar e quantificar tendências nas publicações, criando base comparativa para a realização de inferências, conforme apresentado na seção de resultados. Dessa forma, elementos qualitativos podem complementar os dados quantitativos e fornecer visão mais ampla das contribuições dos trabalhos, conforme descrito por Pluye and Hong (2014).

\section{Quadro 2}

\section{Setores econômicos encontrados}

\begin{tabular}{|c|c|}
\hline Setor econômico & Descrição \\
\hline Academia & $\begin{array}{l}\text { Atividades educacionais voltadas à preparação escolar e universitária, envolvendo } \\
\text { temas como aprendizagem móvel, disponibilidade de conteúdo digital em sala de aula, } \\
\text { ética em pesquisa eletrônica, fábricas de aprendizado e formação de professores. }\end{array}$ \\
\hline Construção Civil & $\begin{array}{l}\text { Temas relacionados ao setor de Construção Civil, tanto na área de projetos quanto na } \\
\text { execução em campo. }\end{array}$ \\
\hline $\begin{array}{l}\text { Desenvolvimento de } \\
\text { Produtos }\end{array}$ & $\begin{array}{l}\text { Operações voltadas à pesquisa e ao desenvolvimento de produtos, principalmente no } \\
\text { contexto de empreendedorismo e startups. }\end{array}$ \\
\hline Energia & $\begin{array}{l}\text { Setor energético, considerando as fontes de energia clássicas não-renováveis e as } \\
\text { inovadoras e limpas, nos contextos de produção e distribuição. }\end{array}$ \\
\hline Manufatura & $\begin{array}{l}\text { Todas as atividades relacionadas diretamente à gestão da produção e à manufatura de } \\
\text { produtos, principalmente em cenário de chão de fábrica. }\end{array}$ \\
\hline Saúde & $\begin{array}{l}\text { Ações voltadas a qualquer tema da área médica, envolvendo tanto tratamento } \\
\text { hospitalar quanto terapia, enfermagem e cuidado com idosos, bem como a busca de } \\
\text { informações referentes à saúde. }\end{array}$ \\
\hline Setor Público & $\begin{array}{l}\text { Temáticas referentes à esfera pública, envolvendo temas como a segurança pública e } \\
\text { a comunicação emergencial com cidadãos. }\end{array}$ \\
\hline Urbanismo & $\begin{array}{l}\text { Atividades direcionadas ao urbanismo, principalmente no contexto de exploração e } \\
\text { incentivo ao sentimento de pertencimento à cidade. }\end{array}$ \\
\hline
\end{tabular}

Fonte: Autores (2021). 


\section{Quadro 3}

Tecnologias e contextos tecnológicos encontrados

\begin{tabular}{|c|c|}
\hline Tecnologia & Descrição \\
\hline Big Data & $\begin{array}{l}\text { Ciência que envolve as atividades de coleta, armazenamento, transformação, } \\
\text { análise e extração de conhecimento de grandes bases de dados. }\end{array}$ \\
\hline $\begin{array}{l}\text { Building Information } \\
\text { Modeling (BIM) }\end{array}$ & $\begin{array}{l}\text { Representação digital de todas as características de uma edificação, considerando } \\
\text { projeto, construção e desempenho, sendo fortemente apoiada em parametrização } \\
\text { de elementos para melhorar a percepção e evitar incompatibilidades. }\end{array}$ \\
\hline Blockchain & $\begin{array}{l}\text { Tecnologia de armazenamento de informações em blocos transacionais que } \\
\text { constituem cadeias. Oferece grande confiabilidade e segurança, sendo muito } \\
\text { utilizada em transações de criptomoedas. }\end{array}$ \\
\hline $\begin{array}{l}\text { Comunicação } \\
\text { Audiovisual }\end{array}$ & $\begin{array}{l}\text { Mídias audiovisuais, de utilização frequente na transformação digital, com destaque } \\
\text { para os vídeos publicados em plataformas como o Youtube. }\end{array}$ \\
\hline Digital Learning & $\begin{array}{l}\text { Conjunto de tecnologias voltadas ao aprendizado, com destaque para as que } \\
\text { compõem o E-Learning, baseado em plataformas para dispositivos móveis. }\end{array}$ \\
\hline $\begin{array}{l}\text { Fábricas de } \\
\text { Aprendizado }\end{array}$ & $\begin{array}{l}\text { Ambientes de simulação organizados em módulos, visando emular o ambiente de } \\
\text { desenvolvimento e produção utilizando tecnologias da Indústria 4.0. }\end{array}$ \\
\hline Gamificação & $\begin{array}{l}\text { Seleção e aplicação de características do design de jogos a outros contextos, } \\
\text { atuando principalmente como ferramenta motivacional que define objetivos. }\end{array}$ \\
\hline $\begin{array}{l}\text { Manufatura Aditiva } \\
\text { (Impressão 3D) }\end{array}$ & $\begin{array}{l}\text { Fabricação de objetos modelados tridimensionalmente em computador, utilizando } \\
\text { processo aditivo de múltiplas camadas de material. Promove, principalmente, a } \\
\text { rápida prototipagem de elementos complexos. }\end{array}$ \\
\hline Mídias Sociais & $\begin{array}{l}\text { Redes digitais para contato, comunicação e compartilhamento de informações } \\
\text { entre pessoas, com destaque para plataformas como Facebook e Linkedln. }\end{array}$ \\
\hline Modelagem 3D & $\begin{array}{l}\text { Criação de modelos tridimensionais em computador, simulando objetos, cenários e } \\
\text { animações. }\end{array}$ \\
\hline Realidade Aumentada & $\begin{array}{l}\text { Interação entre os ambientes real e virtual, com o objetivo de ampliar o } \\
\text { entendimento do usuário enquanto o mantém atento ao mundo real. Teve início } \\
\text { com os códigos QR e é atualmente baseada em programas e sensores. }\end{array}$ \\
\hline $\begin{array}{l}\text { Sistemas Cyber-Físicos } \\
\text { (CPSs) }\end{array}$ & $\begin{array}{l}\text { Sistemas computacionais que realizam a integração entre os diversos elementos de } \\
\text { uma organização, visando o monitoramento e o controle de informações e } \\
\text { máquinas. }\end{array}$ \\
\hline $\begin{array}{l}\text { Tecnologias de } \\
\text { Informação e } \\
\text { Comunicação (TICS) }\end{array}$ & $\begin{array}{l}\text { Conjunto mais geral de diversas tecnologias responsáveis pela circulação de } \\
\text { informações, auxiliando também na comunicação. }\end{array}$ \\
\hline
\end{tabular}

Fonte: Autores (2021).

Primeiramente, a separação dos artigos em termos de setores econômicos (Quadro 2) foi relacionada com os anos de publicação, visando determinar quais áreas da economia estão associadas ao maior número de pesquisas e se há alguma tendência de expansão. Em seguida, as tecnologias e contextos tecnológicos abordados (Quadro 3) foram também estudados em função da data de publicação, buscando identificar os elementos mais importantes e alterações ao longo do tempo. Posteriormente, os dois setores econômicos preponderantes foram explorados em função das tecnologias.

Por fim, as palavras-chave fornecidas pelos autores de todos os artigos foram selecionadas como texto-base para a elaboração de uma nuvem de palavras, a ser apresentada na Figura 3, objetivando-se melhor visualização das temáticas abordadas. Utilizou-se o software Wordle, 
desconsiderando diferenças entre letras maiúsculas e minúsculas, permitindo números e removendo palavras comuns.

\section{Resultados}

Os 48 artigos analisados, considerando anos de publicação, autores e títulos, estão apresentados no Quadro 4.

\section{Quadro 4}

Artigos analisados

\begin{tabular}{|c|c|c|c|}
\hline № & Ano & Autores & Título \\
\hline 1 & 2008 & Höök, K. & $\begin{array}{l}\text { Knowing, Communicating, and Experiencing through Body } \\
\text { and Emotion }\end{array}$ \\
\hline 2 & 2008 & Tian, X., Martin, B., \& Deng, H. & $\begin{array}{l}\text { The impact of digitization on business models for publishing: } \\
\text { Some indicators from a research project }\end{array}$ \\
\hline 3 & 2009 & Benckendorff, P. & $\begin{array}{l}\text { Evaluating Wikis as an assessment tool for developing } \\
\text { collaboration and knowledge management skills }\end{array}$ \\
\hline 4 & 2011 & Mutula, S. M. & Ethics and trust in digital scholarship \\
\hline 5 & 2012 & $\begin{array}{l}\text { Batty, M., Axhausen, K. W., } \\
\text { Giannotti, F., Pozdnoukhov, A., } \\
\text { Bazzani, A., Wachowicz, M., } \\
\text { Ouzounis, G., \& Portugali, Y. }\end{array}$ & Smart cities of the future \\
\hline 6 & 2013 & Cobo, C. & $\begin{array}{l}\text { Exploration of open educational resources in non-english } \\
\text { speaking communities }\end{array}$ \\
\hline 7 & 2013 & Kalman, J., \& Guerrero, E. & $\begin{array}{l}\text { A Social Practice Approach to Understanding Teachers' } \\
\text { Learning to Use Technology and Digital Literacies in the } \\
\text { Classroom }\end{array}$ \\
\hline 8 & 2013 & Leask, M., \& Younie, S. & $\begin{array}{l}\text { National models for continuing professional development: } \\
\text { The challenges of twenty-first-century knowledge } \\
\text { management }\end{array}$ \\
\hline 9 & 2014 & $\begin{array}{l}\text { Hamilton, A. L., Coldwell-Neilson, } \\
\text { J., \& Craig, A. }\end{array}$ & $\begin{array}{l}\text { Development of an information management knowledge } \\
\text { transfer framework for evidence-based occupational therapy }\end{array}$ \\
\hline 10 & 2014 & Pigg, $\mathrm{S}$. & $\begin{array}{l}\text { Coordinating constant invention: Social media's role in } \\
\text { distributed work }\end{array}$ \\
\hline 11 & 2015 & Gan, B., Menkhoff, T., \& Smith, R. & $\begin{array}{l}\text { Enhancing students' learning process through interactive } \\
\text { digital media: New opportunities for collaborative learning }\end{array}$ \\
\hline 12 & 2015 & $\begin{array}{l}\text { Ji, X., Xu, J.-J., Wei, K.-C., \& Tang, } \\
\text { S.-W. }\end{array}$ & New paradigm and key technologies of chemical industry 4.0 \\
\hline 13 & 2015 & $\begin{array}{l}\text { Jones, S.-L., Procter, R., \& Younie, } \\
\text { S. }\end{array}$ & $\begin{array}{l}\text { Participatory knowledge mobilisation: an emerging model for } \\
\text { international translational research in education }\end{array}$ \\
\hline 14 & 2015 & $\begin{array}{l}\text { Limaye, R. J., Deka, S., Ahmed, N., } \\
\text { \& Mwaikambo, L. }\end{array}$ & $\begin{array}{l}\text { Designing elearning courses to meet the digital literacy } \\
\text { needs of healthcare workers in lower-and middle-income } \\
\text { countries: Experiences from the Knowledge for Health } \\
\text { Project }\end{array}$ \\
\hline 15 & 2015 & $\begin{array}{l}\text { López, D., Marulanda, C. E., \& } \\
\text { López, M. }\end{array}$ & $\begin{array}{l}\text { Metrics for assessing knowledge management for small and } \\
\text { medium size companies of the information and technology } \\
\text { sector in the Colombian coffee triangle }\end{array}$ \\
\hline & & & Continua \\
\hline
\end{tabular}




\begin{tabular}{|c|c|c|c|}
\hline № & Ano & Autores & Título \\
\hline 16 & 2016 & $\begin{array}{l}\text { Donoso, V., Verdoodt, V., } \\
\text { Mechelen, M. van, \& Jasmontaite, } \\
\text { L. }\end{array}$ & $\begin{array}{l}\text { Faraway, so close: Why the digital industry needs scholars } \\
\text { and the other way around }\end{array}$ \\
\hline 17 & 2017 & Huang, C.-K., \& Lin, C.-Y. & $\begin{array}{l}\text { Flipping business education: Transformative use of team- } \\
\text { based learning in human resource management classrooms }\end{array}$ \\
\hline 18 & 2017 & Madsen, O., \& Møller, C. & $\begin{array}{l}\text { The AAU Smart Production Laboratory for teaching and } \\
\text { research in emerging digital manufacturing technologies }\end{array}$ \\
\hline 19 & 2017 & $\begin{array}{l}\text { Mola, L., Russo, I., Giangreco, A., } \\
\text { \& Rossignoli, C. }\end{array}$ & $\begin{array}{l}\text { Who knows what? Reconfiguring the governance and the } \\
\text { capabilities of the supply chain between physical and digital } \\
\text { processes in the fashion industry }\end{array}$ \\
\hline 20 & 2017 & $\begin{array}{l}\text { Wang, M., Zheng, M., Tian, L., Qiu, } \\
\text { Z., \& Li, X. }\end{array}$ & $\begin{array}{l}\text { A full life cycle nuclear knowledge management framework } \\
\text { based on digital system }\end{array}$ \\
\hline 21 & 2018 & Deuff, O. L. & umanités digitales: Un cas particulier de \\
\hline 22 & 2018 & $\begin{array}{l}\text { Hannola, L., Richter, A., Richter, } \\
\text { S., \& Stocker, A. }\end{array}$ & $\begin{array}{l}\text { Empowering production workers with digitally facilitated } \\
\text { knowledge processes-a conceptual framework }\end{array}$ \\
\hline 23 & 2018 & Hulin, $\mathrm{T}$. & $\begin{array}{l}\text { De la gestion procédurale des connaissances au management } \\
\text { réflexif: L'exemple de la formation aux usages du numérique }\end{array}$ \\
\hline 24 & 2018 & $\begin{array}{l}\text { Ilvonen, I., Thalmann, S., } \\
\text { Manhart, M., \& Sillaber, C. }\end{array}$ & $\begin{array}{l}\text { Reconciling digital transformation and knowledge protection: } \\
\text { A research agenda }\end{array}$ \\
\hline 25 & 2018 & Jarrahi, & $\begin{array}{l}\text { Social Media, Social Capital, and Knowledge Sharing in } \\
\text { Enterprise }\end{array}$ \\
\hline 26 & 2018 & Mayer, F. & $\begin{array}{l}\text { Exploring the notion of situation for responsive } \\
\text { manufacturing systems specification issues }\end{array}$ \\
\hline 27 & 2018 & Papadonikolaki, E. & $\begin{array}{l}\text { Loosely Coupled Systems of Innovation: Aligning BIM } \\
\text { Adoption with Implementation in Dutch Construction }\end{array}$ \\
\hline 28 & 2018 & Pfouga, A., \& Stiepandić J. & $\begin{array}{l}\text { Leveraging 3D geometric knowledge in the product lifecycle } \\
\text { based on industrial standards }\end{array}$ \\
\hline 29 & 2018 & asidharan, A., \& Janodia, M. D. & $\begin{array}{l}\text { Social Media: A Double Edged Sword for Accessing Health } \\
\text { Care Information }\end{array}$ \\
\hline 30 & 2019 & $\begin{array}{l}\text { Bonanomi, M. M., Hall, D. M., } \\
\text { Staub-French, S., Tucker, A., \& } \\
\text { Talamo, C. M. L. }\end{array}$ & $\begin{array}{l}\text { The impact of digital transformation on formal and informal } \\
\text { organizational structures of large architecture and } \\
\text { engineering firms }\end{array}$ \\
\hline 31 & 2019 & $\begin{array}{l}\text { De Vasconcellos Motta, F. M., } \\
\text { Barbosa, C. R., \& Barbosa, R. R. }\end{array}$ & $\begin{array}{l}\text { Big data as innovation source in museums: The case study of } \\
\text { the British Museum }\end{array}$ \\
\hline 32 & 2019 & $\begin{array}{l}\text { Dele-Ajayi, O., Strachan, R., } \\
\text { Pickard, A. J., \& Sanderson, J. J. }\end{array}$ & $\begin{array}{l}\text { Games for Teaching Mathematics in Nigeria: What Happens } \\
\text { to Pupils' Engagement and Traditional Classroom Dynamics? }\end{array}$ \\
\hline 33 & 2019 & $\begin{array}{l}\text { Draǧoicea, M., Léonard, M., } \\
\text { Ciolofan, S. N., \& Militaru, G. }\end{array}$ & $\begin{array}{l}\text { Managing Data, Information, and Technology in Cyber } \\
\text { Physical Systems: Public Safety as a Service and its Systems }\end{array}$ \\
\hline 34 & 2019 & , P. E., \& & $\begin{array}{l}\text { Live-action Communication Design: A Technical How-To } \\
\text { Video Case Study }\end{array}$ \\
\hline 35 & 2019 & $\begin{array}{l}\text { Hietajärvi, L., Salmela-Aro, K., } \\
\text { Tuominen, H., Hakkarainen, K., \& } \\
\text { Lonka, K. }\end{array}$ & $\begin{array}{l}\text { Beyond screen time: Multidimensionality of socio-digital } \\
\text { participation and relations to academic well-being in three } \\
\text { educational phases }\end{array}$ \\
\hline 36 & 2019 & $\begin{array}{l}\text { Naveed, Q. N., Mohamed } \\
\text { Qureshi, M. R. N., Shaikh, A., } \\
\text { Alsayed, A. O., Sanober, S., \& } \\
\text { Mohiuddin, K. }\end{array}$ & $\begin{array}{l}\text { Evaluating and Ranking Cloud-Based E-Learning Critical } \\
\text { Success Factors (CSFs) Using Combinatorial Approach }\end{array}$ \\
\hline 37 & 2019 & $\begin{array}{l}\text { Pang, C., Neustaedter, C., } \\
\text { Moffatt, K., Hennessy, K., \& Pan, } \\
\text { R. }\end{array}$ & $\begin{array}{l}\text { The role of a location-based city exploration game in digital } \\
\text { placemaking }\end{array}$ \\
\hline 38 & 2019 & $\begin{array}{l}\text { Roberts, C. J., Edwards, D. J., } \\
\text { Hosseini, M. R., Mateo-Garcia, M., } \\
\text { \& Owusu-Manu, D.-G. }\end{array}$ & Post-occupancy evaluation: a review of literature \\
\hline
\end{tabular}




\begin{tabular}{|c|c|c|c|}
\hline № & Ano & Autores & Título \\
\hline 39 & 2019 & Venkitachalam, K., \& Bosua, R. & $\begin{array}{l}\text { Perspectives on effective digital content management in } \\
\text { organizations }\end{array}$ \\
\hline 40 & 2020 & Bouncken, R., \& Barwinski, R. & $\begin{array}{l}\text { Shared digital identity and rich knowledge ties in global 3D } \\
\text { printing - A drizzle in the clouds? }\end{array}$ \\
\hline 41 & 2020 & $\begin{array}{l}\text { Caldarelli, G., Rossignoli, C., \& } \\
\text { Zardini, A. }\end{array}$ & $\begin{array}{l}\text { Overcoming the blockchain oracle problem in the traceability } \\
\text { of non-fungible products }\end{array}$ \\
\hline 42 & 2020 & $\begin{array}{l}\text { Kamalaldin, A., Linde, L., Sjödin, } \\
\text { D., \& Parida, V. }\end{array}$ & $\begin{array}{l}\text { Transforming provider-customer relationships in digital } \\
\text { servitization: A relational view on digitalization }\end{array}$ \\
\hline 43 & 2020 & Li, Y-W., \& Cao, K. & $\begin{array}{l}\text { Establishment and application of intelligent city building } \\
\text { information model based on BP neural network model }\end{array}$ \\
\hline 44 & 2020 & Lindvig, K., \& Mathiasen, H. & $\begin{array}{l}\text { Translating the Learning Factory model to a Danish } \\
\text { Vocational Education Setting }\end{array}$ \\
\hline 45 & 2020 & Thanachawengsakul, N. & $\begin{array}{l}\text { A conceptual framework for the development of a moocs- } \\
\text { based knowledge repository to enhance digital } \\
\text { entrepreneurs' competencies }\end{array}$ \\
\hline 46 & 2020 & $\begin{array}{l}\text { Tortorella, G. L., Cawley Vergara, } \\
\text { A. M., Garza-Reyes, J. A., \& } \\
\text { Sawhney, R. }\end{array}$ & $\begin{array}{l}\text { Organizational learning paths based upon industry } 4.0 \\
\text { adoption: An empirical study with Brazilian manufacturers }\end{array}$ \\
\hline 47 & 2020 & Tsertsidis, A. & $\begin{array}{l}\text { Challenges in the provision of digital technologies to elderly } \\
\text { with dementia to support ageing in place: a case study of a } \\
\text { Swedish municipality }\end{array}$ \\
\hline 48 & 2020 & $\begin{array}{l}\text { Zangiacomi, A., Pessot, E., } \\
\text { Fornasiero, R., Bertetti, M., \& } \\
\text { Sacco, M. }\end{array}$ & $\begin{array}{l}\text { Moving towards digitalization: a multiple case study in } \\
\text { manufacturing }\end{array}$ \\
\hline
\end{tabular}

Fonte: Autores (2021).

O resultado da categorização de artigos por setor econômico, considerando os anos de publicação, está apresentado na Figura 1.

Figura 1

Artigos publicados por setores econômicos em função do ano

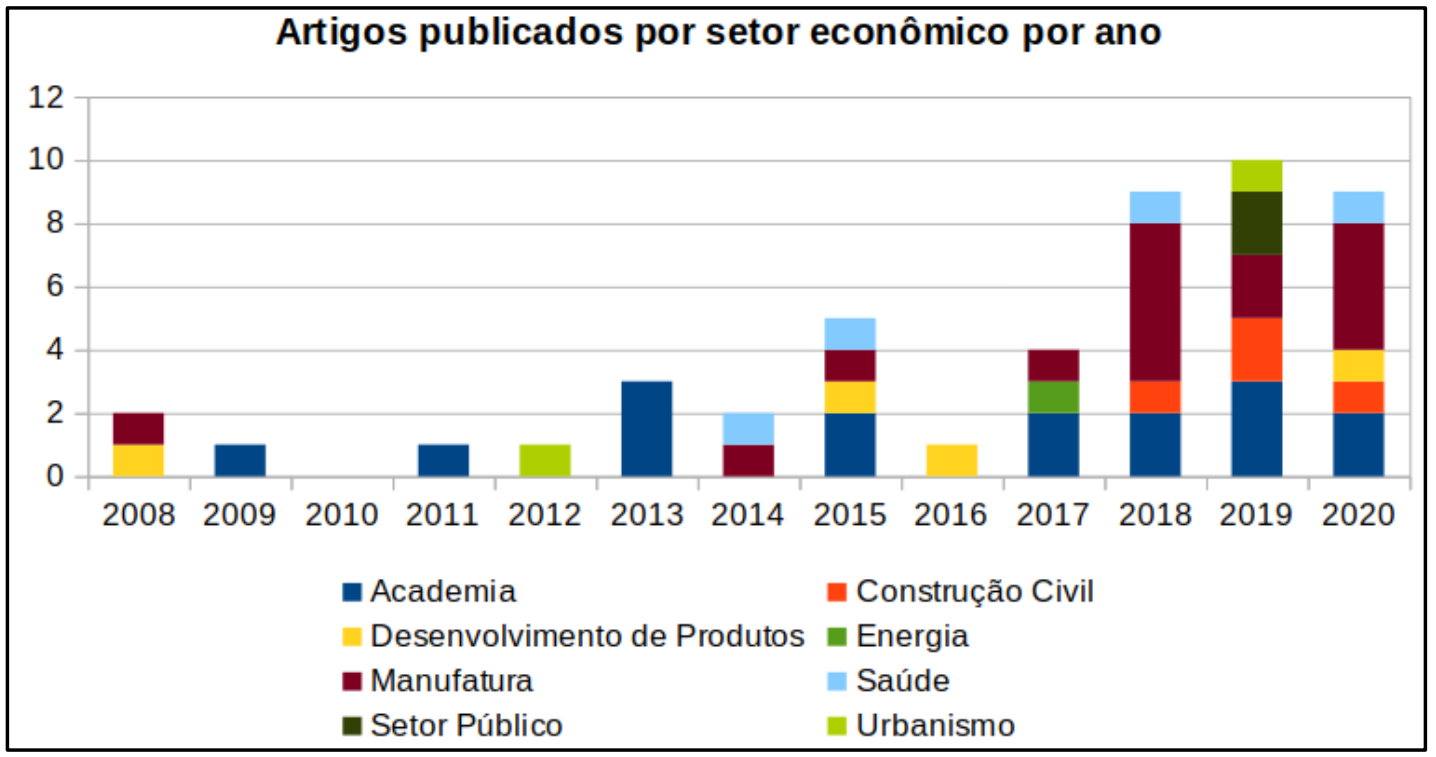

Fonte: Autores (2021). 
Conforme demonstrado na Figura 1, o número de publicações sobre a temática manteve-se baixo entre os anos de 2008 a 2012, variando de nenhum a dois artigos publicados por ano. Em 2013, ocorreu o primeiro salto numérico, quando três documentos foram publicados. Cabe ressaltar que este é o ano do lançamento da Plataforma Industrie 4.0 (Kagermann et al., 2016), o que pode ter contribuído para aumentar a visibilidade da transformação digital. Entre os anos de 2014 e 2017, o número de publicações apresentou variação instável, com destaque para o crescimento em 2015, quando 5 trabalhos foram publicados. Apesar da queda em 2016, pode-se inferir que já existia aumento de interesse. Os anos de 2018, 2019 e 2020 foram marcados por grande número de publicações, uma vez que a maior digitalização e o acirramento da competitividade no mercado tendem a estimular o desenvolvimento de pesquisas. Cabe ressaltar, ainda, que o ano de 2020 foi contabilizado apenas até o início do mês de maio. Comprova-se, portanto, a primeira hipótese.

A Figura 1 indica que o setor acadêmico, com 16 publicações (aproximadamente 33,3\%), apresenta-se como o mais frequente. Essa constatação vai de encontro à tendência anunciada por Toffler (1990) e Drucker (1994) e cada vez mais pronunciada na sociedade moderna: o conhecimento passa a atuar como a matéria-prima mais importante das organizações. Naturalmente, a academia busca, constantemente, desenvolver cursos mais atrativos para o contexto industrial, formando profissionais mais flexíveis e adaptados às novas competências. Essa movimentação coincide com o princípio de criação de valor em toda a cadeia de fornecimento (Revilla \& Knoppen, 2015), pois a academia está - ou deveria estar - intimamente conectada às atividades industriais. A segunda hipótese foi confirmada.

Apesar da maioria das publicações ser referente ao setor acadêmico, a manufatura foi representada por proporção semelhante: 15 artigos (aproximadamente 31,3\%). As atividades industriais manufatureiras representam a "linha de frente" da economia, intensamente afetada pela transformação digital, e é esperado que sejam referenciadas por diversas pesquisas sobre a temática, pois a sobrevivência dessas empresas depende diretamente da capacidade de absorver e aplicar conhecimentos, conforme apontado por Belluzzo e Feres (2009). O acirramento da competitividade é responsável por intensificar as atividades de GC dentro dessas organizações, algo reforçado pela presença de maior número de publicações a partir de 2018, enquanto trabalhos voltados à academia estão distribuídos de forma mais homogênea ao longo do tempo. Infere-se, portanto, que a terceira hipótese foi comprovada.

Com relação aos demais setores, a Construção Civil, o desenvolvimento de produtos e a saúde foram representados por quatro artigos cada (aproximadamente 8,3\%). Para a Construção Civil, a transformação digital é importante principalmente por promover, através de sistemas BIM, a comunicação e a compatibilização de distintos projetos, evitando incompatibilidades. Para o desenvolvimento de produtos, as tecnologias permitem encurtar o tempo de prototipagem e 
lançamento, contribuindo também para a criação de Produtos Mínimos Viáveis (Minimum Viable Products - MVPs) mais realistas e, ao mesmo tempo, baratos. Para a saúde, as principais preocupações giram em torno da circulação e obtenção de informações médicas pela população, bem como a atualização de conhecimentos de médicos, enfermeiros e cuidadores.

O setor público e o urbanismo foram referenciados por apenas dois artigos cada (aproximadamente 4,2\%) e o setor de energia estava envolvido em somente um documento (2,1\%). A transformação digital não deixa, contudo, de ter impactos relevantes: para os dois primeiros, a comunicação com a população, a transparência e a disseminação de conhecimentos sobre a infraestrutura urbana são favorecidos, enquanto o último é beneficiado pelo melhor controle informacional de redes de produção e distribuição complexas e cercadas de riscos. Por fim, cabe ressaltar que não foram encontrados trabalhos voltados diretamente ao setor de serviços, que também é impactado pelo contexto da Indústria 4.0: a área ainda é, infelizmente, pouco considerada ao abordar a GC para a Quarta Revolução Industrial, conforme apontado por Shamim, Cang, Yu and Li (2017).

As ocorrências de principais tecnologias e contextos tecnológicos nos artigos estão apresentadas na Tabela 1 . O predomínio de pesquisas voltadas aos setores acadêmico e de manufatura é refletido nos contextos tecnológicos. Fica evidente a importância das tecnologias de informação e comunicação que, conforme apontado por Lenz et al. (2018), são o mais importante pilar da Quarta Revolução Industrial. Essas tecnologias estão representadas em 14 artigos (aproximadamente 29,2\%) e são referenciadas principalmente por estudos voltados à manufatura, que tratam fundamentalmente de questões de circulação de dados no ambiente produtivo. Contudo, o contexto de Digital Learning, associado sobretudo ao meio acadêmico e presente em outros 14 trabalhos (29,2\%), também contempla diversas tecnologias que, direta ou indiretamente, estão associadas às TICs, como a aprendizagem por dispositivos móveis e a criação de plataformas para armazenamento e disponibilização de conteúdo digital. Comprova-se, portanto, a quarta hipótese. 
Tabela 1

Tecnologias e contextos tecnológicos em função dos anos de publicação

\begin{tabular}{|c|c|c|c|c|c|c|c|c|}
\hline $\begin{array}{c}\text { Tecnologias e contextos } \\
\text { Tecnológicos } \\
\end{array}$ & $\begin{array}{c}2008 \text { a } \\
2011 \\
\end{array}$ & $\begin{array}{c}2012 a \\
2015 \\
\end{array}$ & 2016 & 2017 & 2018 & 2019 & 2020 & Total \\
\hline Big Data & & & & & & 1 & & 1 \\
\hline Building Information Modeling & & & & & 1 & 1 & 1 & 3 \\
\hline Blockchain & & & & & & & 1 & 1 \\
\hline Comunicação Audiovisual & & & & & & 1 & & 1 \\
\hline Digital Learning & 2 & 6 & & 1 & 2 & 1 & 2 & 14 \\
\hline Fábricas de Aprendizado & & & & 1 & & & 1 & 2 \\
\hline Gamificação & & & & & & 2 & & 2 \\
\hline Impressão 3D & & & & & & & 1 & 1 \\
\hline Mídias Sociais & & 1 & & & 2 & 1 & & 4 \\
\hline Modelagem 3D & & & & & 1 & & & 1 \\
\hline Realidade Aumentada & 1 & & & & & & & 1 \\
\hline Sistemas Cyber-Físicos & & 1 & & & 1 & 1 & & 3 \\
\hline Tec. Informação e Comunicação & 1 & 3 & 1 & 2 & 2 & 2 & 3 & 14 \\
\hline Total & 4 & 11 & 1 & 4 & 9 & 10 & 9 & 48 \\
\hline
\end{tabular}

Fonte: Autores (2021).

Com relação às demais tecnologias, conforme a Tabela 1, as mídias sociais aparecem em terceiro lugar, sendo representadas em quatro artigos (aproximadamente 8,3\%). A utilização desses meios é consequência direta das TICs e resulta, simultaneamente, em efeitos positivos e negativos: se, por um lado, as mídias sociais podem atuar como importantes plataformas de networking e compartilhamento de conhecimento, podem também facilitar a circulação de informações e notícias falsas e promover distrações no ambiente de trabalho. Os Sistemas Cyber-Físicos, que dependem das TICs para administrar informações, e o BIM, diretamente relacionado à constante integração de projetos e projetistas, foram mencionados em três trabalhos cada (6,3\%). As Fábricas de Aprendizado e a Gamificação, importantes visões inovadoras para aperfeiçoar o aprendizado, foram representadas por dois artigos cada (4,2\%). As demais tecnologias estiveram associadas a apenas um trabalho cada $(2,1 \%)$.

É interessante destacar que, antes do ano de 2017, apenas o Digital Learning, as TICs, as mídias sociais, os Sistemas Cyber-Físicos e a Realidade Aumentada estavam presentes na amostra. Conceitos como Big Data, Blockchain, Impressão 3D - e, consequentemente, Modelagem 3D - e a visão da Gamificação tornaram-se mais populares apenas recentemente, o que poderia explicar a representação somente após esse período.

A análise de tecnologias, considerando apenas os setores acadêmico e de manufatura, encontra-se na Figura 2. 
Figura 2

Contextos tecnológicos da academia e da manufatura

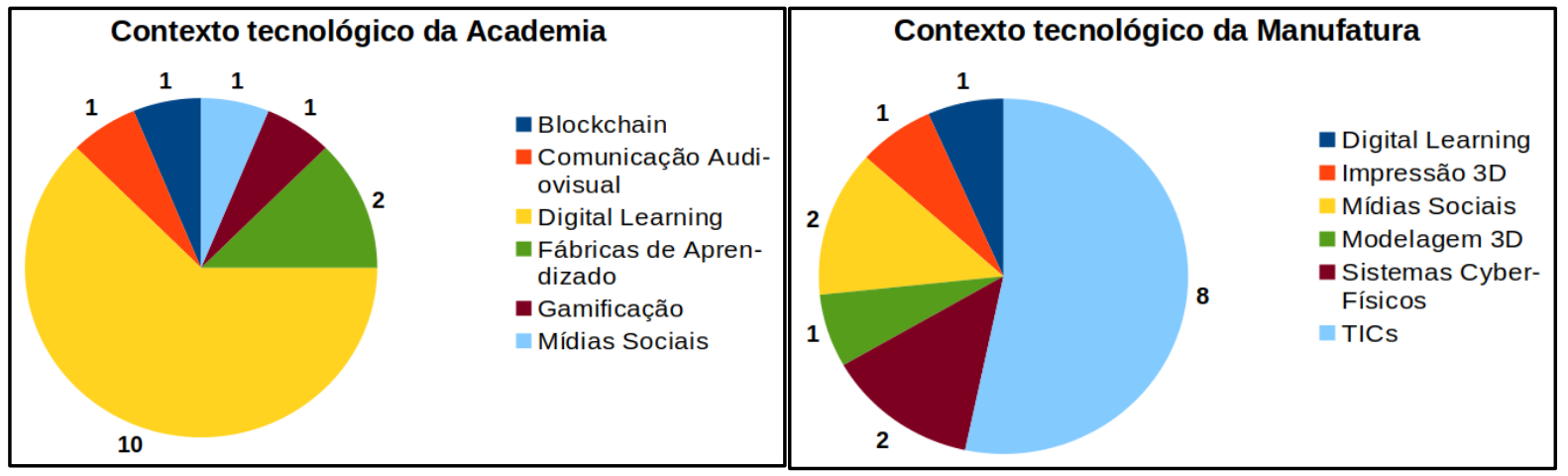

Fonte: Autores (2021).

A Figura 2 indica um direcionamento tecnológico para cada setor. A academia prioriza formas inovadoras de desenvolver o aprendizado e, portanto, busca abordar conceitos como as Fábricas de Aprendizado e a Gamificação em apoio ao Digital Learning. O contexto acadêmico acaba por envolver, também, preocupações com a comunicação audiovisual e as mídias sociais. O Blockchain, por sua vez, foi referenciado por um artigo que tratava principalmente da dificuldade de abordagem do problema do oráculo na literatura (no 41). Com relação à manufatura, além das TICs, os Sistemas Cyber-Físicos, a Modelagem 3D e a Impressão 3D são diretamente influentes nas atividades de produção, o que justifica sua presença nos trabalhos. O Digital Learning e as mídias sociais, por atuarem nas relações de comunicação e aprendizado entre funcionários, também estiveram presentes. Infere-se, portanto, que há comprovação da quinta e última hipótese para esses setores.

A nuvem de palavras, criada a partir das palavras-chave fornecidas pelos autores, está apresentada na Figura 3. A busca utilizou construtos relacionados à transformação digital e à GC e, portanto, há predominância dos termos "knowledge", "management" e "digital", como esperado. A forte presença do setor acadêmico é ressaltada por termos como "learning" e "education", que também aparecem continuamente, complementados por expressões menos frequentes, porém geralmente associadas, como "studies", "research", "teaching" e "literacy". Impactos da transformação digital podem ter condicionado o surgimento de palavras como "transformation", "development", "innovation" e "empowerment".

A manufatura pode ser representada por palavras como "industry", "supply", "chain" e "business", complementadas por "manufacturing" e "production". A importância das TICs pode ser associada a termos constantes como "information" e "technologies", assim como a expressões menos frequentes, como "network(s)" e "system(s)". Por fim, vale ressaltar a presença de expressões que 
indicam relação com o compartilhamento do conhecimento, como "social", "transfer", "organizational", "interaction", "collaboration" e "communication".

Figura 3

Nuvem de palavras elaborada a partir das palavras-chave dos artigos

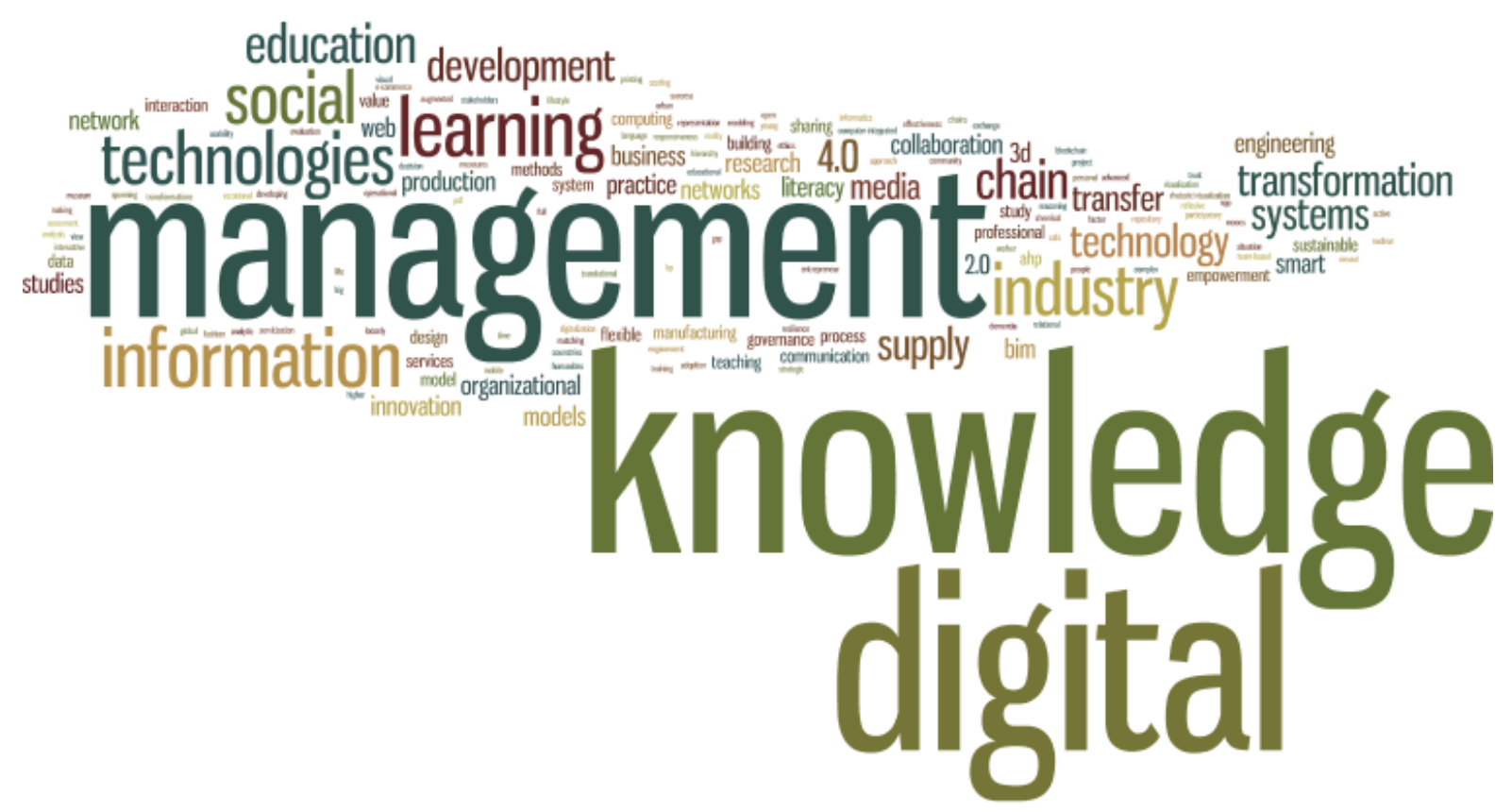

Fonte: Autores (2021).

\section{Considerações Finais}

Diante da grande pressão exercida pela transformação digital sobre as relações tradicionais de mercado, acirrando a competitividade, é essencial compreender quais tecnologias têm recebido destaque e onde estão sendo aplicadas. Este artigo aponta setores econômicos e tecnologias digitais que podem ser especialmente interessantes para a gestão e o compartilhamento do conhecimento diante desse contexto. Destaca, também, a importância de promover pesquisas nessas áreas, uma vez que a inovação, elemento muitas vezes desprezado, torna-se cada vez mais essencial para a sobrevivência das organizações e evidencia-se como competência de interesse na academia.

Organizações que realizam atividades associadas aos setores apontados neste trabalho devem priorizar investimentos em algumas tecnologias digitais para que mantenham a competividade. O setor acadêmico, por sua vez, deve abordar os novos conhecimentos necessários para a operação dessas tecnologias e adotar estratégias como o Digital Learning e as Fábricas de Aprendizado. Por fim, cabe ao governo direcionar políticas públicas para favorecer o desenvolvimento de um ambiente propício para a Quarta Revolução Industrial. 
Considera-se que os objetivos foram atingidos. As três primeiras hipóteses foram confirmadas ao interpretar a Figura 1 e as duas últimas foram verificadas ao analisar conjuntamente a Tabela 1 e a Figura 2. Como possível limitação deste artigo, cabe citar o reduzido número de publicações analisadas, dada a contemporaneidade do tema, o que pode condicionar menor aderência dos dados à realidade. Sabendo-se que o número de artigos publicados tende a ser ampliado de forma muito acelerada, novas pesquisas podem ser realizadas futuramente, adicionando novos trabalhos, para que a evolução temporal dos dados seja analisada.

\section{Referências}

Araújo, A. G., Carneiro, A. M. P., \& Palha, R. P. (2020). Sustainable construction management: A systematic review of the literature with meta-analysis. Journal of Cleaner Production, 256. https://doi.org/10.1016/j.jclepro.2020.120350

Batista, F. F. (2012). Modelo de gestão do conhecimento para a administração pública brasileira: como implementar a gestão do conhecimento para produzir resultados em benefício do cidadão. Recuperado em 15 dezembro, 2019, de: https://bit.ly/2Z3IHCO

Batty, M., Axhausen, K. W., Giannotti, F., Pozdnoukhov, A., Bazzani, A., Wachowicz, M., Ouzounis, G., \& Portugali, Y. (2012). Smart cities of the future. European Physical Journal: Special Topics, 214(1), 481-518. https://doi.org/10.1140/epjst/e2012-01703-3

Belluzzo, R. C. B., \& Feres, G. G. (2009). Competência em informação: um diferencial da qualidade em publicações científicas. Revista Brasileira de Biblioteconomia e Documentação - RBBD, 5(2), 70-83. Recuperado em 10 de abril, 2020, de: https://rbbd.febab.org.br/rbbd/article/download/143/149

Benckendorff, P. (2009). Evaluating Wikis as an assessment tool for developing collaboration and knowledge management skills. Journal of Hospitality and Tourism Management, 16(1), 102-

\section{2. https://doi.org/10.1375/jhtm.16.1.102}

Bonanomi, M. M., Hall, D. M., Staub-French, S., Tucker, A., \& Talamo, C. M. L. (2019). The impact of digital transformation on formal and informal organizational structures of large architecture and engineering firms. Engineering, Construction and Architectural Management, 27(4), 872- 
Bouncken, R., \& Barwinski, R. (2020). Shared digital identity and rich knowledge ties in global 3D printing - A drizzle in the clouds? Global Strategy Journal, 28 pp. https://doi.org/10.1002/gsj.1370

Caldarelli, G., Rossingnoli, C., \& Zardini, A. (2020). Overcoming the blockchain oracle problem in the traceability of non-fungible products. Sustainability (Switzerland), 12(6), $17 \mathrm{pp}$. https://doi.org/10.3390/su12062391

Centenaro, A., Bonemberger, A. M. O., \& Laimer, C. G. (2016). Gestão do conhecimento e vantagem competitiva: estudo no setor metalmecânico. Revista de Ciências da Administração, 18(44), 38-51. http://dx.doi.org/10.5007/2175-8077.2016v18n44p38

Choo, C. W. (2003). A organização do conhecimento: como as organizações usam a informação para criar significado, construir conhecimento e tomar decisões. São Paulo: Senac São Paulo.

Cobo, C. (2013). Exploration of open educational resources in non-english speaking communities. International Review of Research in Open and Distance Learning, 14(2), 106-128. https://doi.org/10.19173/irrodl.v14i2.1493

Davenport, T. H., \& Prusak, L. (2003). Conhecimento empresarial: como as organizações gerenciam o seu capital intelectual. 237 p. Rio de Janeiro: Elsevier.

De Vasconcellos Motta, F. M., Barbosa, C. R., \& Barbosa, R. R. (2019). Big data como fonte de inovação em museus: O estudo de caso do Museu Britânico. Informação e Sociedade, 29(1), 83-100. Recupeado 10 de abril, 2020, de: https://periodicos.ufpb.br/ojs2/index.php/ies/article/view/44005

Dele-Ajayi, O., Strachan, R., Pickard, A. J., \& Sanderson, J. J. (2019). Games for Teaching Mathematics in Nigeria: What Happens to Pupils' Engagement and Traditional Classroom Dynamics? IEEE Access, 7, 53248-53261. https://doi.org/10.1109/ACCESS.2019.2912359

Deuff, O. L. (2018). Le chercheur en humanités digitales: Un cas particulier de travailleur du savoir? Communication et Management, 14(1), 55-69. https://doi.org/10.3917/comma.141.0055

Donoso, V., Verdoodt, V., Mechelen, M. van, \& Jasmontaite, L. (2016). Faraway, so close: Why the 
digital industry needs scholars and the other way around. Journal of Children and Media, 10(2), 200-207. https://doi.org/10.1080/17482798.2015.1131728

Draǧoicea, M., Léonard, M., Ciolofan, S. N., \& Militaru, G. (2019). Managing Data, Information, and Technology in Cyber Physical Systems: Public Safety as a Service and its Systems. IEEE Access, 7, 92672-92692. https://doi.org/10.1109/ACCESS.2019.2927398

Drucker, P. F. (1994). Sociedade pós-capitalista. São Paulo: Pioneira.

Eriksson, P. E., \& Eriksson, Y. (2019). Live-action Communication Design: A Technical How-To Video Case Study. Technical Communication Quarterly, 28(1), 69-91. https://doi.org/10.1080/10572252.2018.1528388

Gan, B., Menkhoff, T., \& Smith, R. (2015). Enhancing students' learning process through interactive digital media: New opportunities for collaborative learning. Computers in Human Behavior, 51, 652-663. https://doi.org/10.1016/j.chb.2014.12.048

Gölzer, P., \& Fritzsche, A. (2017). Data-driven operations management: organisational implications of the digital transformation in industrial practice. Production Planning \& Control, 28(16), 13321343. https://doi.org/10.1080/09537287.2017.1375148

Gorecky, D., Khamis, M., \& Mura, K. (2017). Introduction and establishment of virtual training in the factory of the future. International Journal of Computer Integrated Manufacturing, 30(1), 182190. https://doi.org/10.1080/0951192X.2015.1067918

Hamilton, A. L., Coldwell-Neilson, J., \& Craig, A. (2014). Development of an information management knowledge transfer framework for evidence-based occupational therapy. VINE, 44(1), 59-93. https://doi.org/10.1108/VINE-12-2012-0051

Hannola, L., Richter, A., Richter, S., \& Stocker, A. (2018). Empowering production workers with digitally facilitated knowledge processes-a conceptual framework. International Journal of Production Research, 56(14), 4729-4743. https://doi.org/10.1080/00207543.2018.1445877

Hietajärvi, L., Salmela-Aro, K., Tuominen, H., Hakkarainen, K., \& Lonka, K. (2019). Beyond screen time: Multidimensionality of socio-digital participation and relations to academic well-being in three 
educational phases. Computers in Human Behavior, 93, 13-24.

https://doi.org/10.1016/j.chb.2018.11.049

Höök, K. (2008). Knowing, Communicating, and Experiencing through Body and Emotion. IEEE Transactions on Learning Technologies, 1(4), 248-259. https://doi.org/10.1109/TLT.2009.3

Huang, C.-K., \& Lin, C.-Y. (2017). Flipping business education: Transformative use of team-based learning in human resource management classrooms. Educational Technology and Society, 20(1), 323-336. Recuperado em 10 de abril, 2020, de: https://bit.ly/3qMt0g6

Hulin, T. (2018). De la gestion procédurale des connaissances au management réflexif: L'exemple de la formation aux usages du numérique. Communication et Management, 14(1), 89-105. https://doi.org/10.3917/comma.141.0089

Ilvonen, I., Thalmann, S., Manhart, M., \& Sillaber, C. (2018). Reconciling digital transformation and knowledge protection: A research agenda. Knowledge Management Research and Practice, 16(2), 235-244. https://doi.org/10.1080/14778238.2018.1445427

Jarrahi, M. H. (2018). Social Media, Social Capital, and Knowledge Sharing in Enterprise. IT Professional, 20(4), 34-45. https://doi.org/10.1109/MITP.2017.265105759

Ji, X., Xu, J.-J., Wei, K.-C., \& Tang, S.-W. (2015). New paradigm and key technologies of chemical industry 4.0. Gao Xiao Hua Xue Gong Cheng Xue Bao/Journal of Chemical Engineering of Chinese Universities, 29(5), 1215-1223. https://doi.org/10.3969/j.issn.1003-9015.2015.05.028 Jones, S.-L., Procter, R., \& Younie, S. (2015). Participatory knowledge mobilisation: an emerging model for international translational research in education. Journal of Education for Teaching, 41(5), 555-573. https://doi.org/10.1080/02607476.2015.1105540

Kagermann, H., Anderl, R., Gausemeier, J., Schuh, G., \& Wahlster, W. (Eds.). (2016). Industrie 4.0 in a Global Context: Strategies for Cooperating with International Partners (acatech STUDY). Munique: Herbert Utz Verlag. Recuperado em 20 de junho, 2019, de: https://bit.ly/374TWjw Kalman, J., \& Guerrero, E. (2013). A Social Practice Approach to Understanding Teachers' Learning to Use Technology and Digital Literacies in the Classroom. E-Learning and Digital Media, 10(3), 
260-275. https://doi.org/10.2304/elea.2013.10.3.260

Kamalaldin, A., Linde, L., Sjödin, D., \& Parida, V. (2020). Transforming provider-customer relationships in digital servitization: A relational view on digitalization. Industrial Marketing Management, 20 pp. https://doi.org/10.1016/j.indmarman.2020.02.004

Klitou, D., Conrads, J., \& Rasmussen, M. (Eds.). (2017). Digital Transformation Monitor: Germany: Industrie 4.0. European Union. Recuperado 20 de abril, 2020, de: https://bit.ly/3oyqbxo

Leask, M., \& Younie, S. (2013). National models for continuing professional development: The challenges of twenty-first-century knowledge management. Professional Development in Education, 39(2), 273-287. https://doi.org/10.1080/19415257.2012.749801

Lenz, J., Wuest, T., \& Westkämper, E. (2018). Holistic approach to machine tool data analytics. Journal of Manufacturing Systems, 48, 180-191. https://doi.org/10.1016/j.jmsy.2018.03.003

Li, Y-W., \& Cao, K. (2020). Establishment and application of intelligent city building information model based on BP neural network model. Computer Communications, 153, 382-389. https://doi.org/10.1016/j.comcom.2020.02.013

Limaye, R. J., Deka, S., Ahmed, N., \& Mwaikambo, L. (2015). Designing eLearning courses to meet the digital literacy needs of healthcare workers in lower- and middle-income countries: Experiences from the Knowledge for Health Project. Knowledge Management and E-Learning, 7(4), 601-615. https://doi.org/10.34105/j.kmel.2015.07.039

Lindvig, K., \& Mathiasen, H. (2020). Translating the Learning Factory model to a Danish Vocational Education Setting. Procedia Manufacturing, 45, 90-95. https://doi.org/10.1016/j.promfg.2020.04.077

López, D., Marulanda, C. E., \& López, M. (2015). Métricas de valoración de la gestión del conocimiento para las pequeñas y medianas empresas del sector tecnologías de información en el triángulo del café en Colombia. Informacion Tecnologica, 26(3), 173-183.

http://dx.doi.org/10.4067/S0718-07642015000300020

Madsen, O., \& Moller, C. (2017). The AAU Smart Production Laboratory for teaching and research in 
emerging digital manufacturing technologies. Procedia Manufacturing, 9, 106-112. https://doi.org/10.1016/j.promfg.2017.04.036

Maglio, M. (2021). Innovation Systems in the Fourth Industrial Revolution: The Territorial Challenge of the Campania Region. In: Bevilacqua C., Calabrò, F., \& Della Spina, L. (Eds). New Metropolitan Perspectives. Smart Innovation, Systems and Technologies, 178, 663-675. Cham: Springer. https://doi.org/10.1007/978-3-030-48279-4_62

Mayer, F. (2018). Exploring the notion of situation for responsive manufacturing systems specification issues. IFAC-PapersOnLine, 51(11), 1579-1585. https://doi.org/10.1016/j.ifacol.2018.08.271

Mola, L., Russo, I., Giangreco, A., \& Rossignoli, C. (2017). Who knows what? Reconfiguring the governance and the capabilities of the supply chain between physical and digital processes in the fashion industry. Production Planning and Control, 28(16), 1284-1297. https://doi.org/10.1080/09537287.2017.1375147

Mutula, S. M. (2011). Ethics and trust in digital scholarship. Electronic Library, 29(2), 261-276. https://doi.org/10.1108/02640471111125212

Naveed, Q. N., Mohamed Qureshi, M. R. N., Shaikh, A., Alsayed, A. O., Sanober, S., \& Mohiuddin, K. (2019). Evaluating and Ranking Cloud-Based E-Learning Critical Success Factors (CSFs) Using Combinatorial Approach. IEEE Access, 7, 157145-157157. https://doi.org/10.1109/ACCESS.2019.2949044

Nonaka, I., \& Takeuchi, H. (1997). Criação de conhecimento na empresa. Rio de Janeiro: Campus.

Pang, C., Neustaedter, C., Moffatt, K., Hennessy, K., \& Pan, R. (2019). The role of a location-based city exploration game in digital placemaking. Behaviour and Information Technology, 25 pp. https://doi.org/10.1080/0144929X.2019.1697899

Papadonikolaki, E. (2018). Loosely Coupled Systems of Innovation: Aligning BIM Adoption with Implementation in Dutch Construction. Journal of Management in Engineering, 34(6), 13 pp. https://doi.org/10.1061/(ASCE)ME.1943-5479.0000644

Pfouga, A., \& Stiepandić, J. (2018). Leveraging 3D geometric knowledge in the product lifecycle based 
on industrial standards. Journal of Computational Design and Engineering, 5(1), 54-67.

https://doi.org/10.1016/j.jcde.2017.11.002

Pigg, S. (2014). Coordinating constant invention: Social media's role in distributed work. Technical Communication Quarterly, 23(2), 69-87. https://doi.org/10.1080/10572252.2013.796545

Pluye, P., \& Hong, Q. N. (2014). Combining the Power of Stories and the Power of Numbers: Mixed Methods Research and Mixed Studies Reviews. Annual Review of Public Health, 35, $29-45$. https://doi.org/10.1146/annurev-publhealth-032013-182440

Polat, L., \& Erkollar, A. (2021). Industry 4.0 vs Society 5.0. In: Durakbasa, N. M., \& Gencyilmaz, M. G. (Eds). Digital Conversion on the Way to Industry 4.0, 333-345. Cham: Springer. https://doi.org/10.1007/978-3-030-62784-3_28

Poloskov, S., Zheltenkov, A., Braga, I., \& Kuznetsova, I. (2020). Adaptation of high-tech knowledgeintensive enterprises to the challenges of Industry 4.0. E3S Web of Conferences, 210, $10 \mathrm{p}$. https://doi.org/10.1051/e3sconf/202021013026

Revilla, E., \& Knoppen, D. (2015). Building knowledge integration in buyer-supplier relationships: The critical role of strategic supply management and trust. International Journal of Operations \& Production Management, 35(10), 1408-1436. https://doi.org/10.1108/IJOPM-01-2014-0030

Ribeiro, J. S. de A. N., França, R., Corrêa, F., \& Ziviani, F. (2019, novembro). Criação de valor para Indústria 4.0: desafios e oportunidades para gestão do conhecimento e tecnologia da informação. Anais do Congresso Internacional de Conhecimento e Inovação - CIKI, Porto Alegre, RS, Brasil, 9. Recuperado em 10 de abril, 2020, de: https://proceeding.ciki.ufsc.br/index.php/ciki/article/view/693

Roberts, C. J., Edwards, D. J., Hosseini, M. R., Mateo-Garcia, M., \& Owusu-Manu, D.-G. (2019). Postoccupancy evaluation: a review of literature. Engineering, Construction and Architectural Management, 26(9), 2084-2106. https://doi.org/10.1108/ECAM-09-2018-0390

Rodrigues, A. R., Tavar, C., Nogueira, G. M., \& Librelotto, R. F. (2016). A bibliometria como ferramenta de análise da produção intelectual: uma análise dos hot topics sobre sustentabilidade. 
Biblionline, 12(3), 34-47. Recuperado em 10 de abril, 2020, de: https://brapci.inf.br/_repositorio/2016/12/pdf_280857d937_0000022127.pdf

Sasidharan, A., \& Janodia, M. D. (2018). Social Media: A Double Edged Sword for Accessing Health Care Information. Indian Journal of Pharmaceutical Education and Research, 52(2), 207-211. https://doi.org/10.5530/ijper.52.2.23

Shamim, S., Cang, S., Yu, H., \& Li, Y. (2017). Examining the feasibilities of Industry 4.0 for the hospitality sector with the lens of management practice. Energies, 10(4). https://doi.org/10.3390/en10040499

Thanachawengsakul, N. (2020). A conceptual framework for the development of a moocs-based knowledge repository to enhance digital entrepreneurs' competencies. International Journal of Information and Education Technology, 10(5), 346-350. https://doi.org/10.18178/ijiet.2020.10.5.1387

Tian, X., Martin, B., \& Deng, H. (2008). The impact of digitization on business models for publishing: Some indicators from a research Project. Journal of Systems and Information Technology, 10(3), 232-250. https://doi.org/10.1108/13287260810916934

Toffler, A. (1990). Powershift: Conhecimento. Riqueza e Violência na borda do Século XXI.

Tortorella, G. L., Cawley Vergara, A. M., Garza-Reyes, J. A., \& Sawhney, R. (2020). Organizational learning paths based upon industry 4.0 adoption: An empirical study with Brazilian manufacturers. International Journal of Production Economics, 219, 284-294. https://doi.org/10.1016/j.ijpe.2019.06.023

Tsertsidis, A. (2020). Challenges in the provision of digital technologies to elderly with dementia to support ageing in place: a case study of a Swedish municipality. Disability and Rehabilitation: Assistive Technology, 12 pp. https://doi.org/10.1080/17483107.2019.1710774

Venkitachalam, K., \& Bosua, R. (2019). Perspectives on effective digital content management in organizations. Knowledge and Process Management, 8 pp. https://doi.org/10.1002/kpm.1600

Wang, M., Zheng, M., Tian, L., Qiu, Z., \& Li, X. (2017). A full life cycle nuclear knowledge management 
framework based on digital system. Annals of Nuclear Energy, 108, 386-393.

https://doi.org/10.1016/j.anucene.2017.04.047

Zangiacomi, A., Pessot, E., Fornasiero, R., Bertetti, M., \& Sacco, M. (2020). Moving towards

digitalization: a multiple case study in manufacturing. Production Planning and Control, 31, 143-157. https://doi.org/10.1080/09537287.2019.1631468 